\title{
Multiprong control of glioblastoma multiforme invasiveness: blockade of pro-inflammatory signaling, anti-angiogenesis, and homeostasis restoration
}

\author{
Nicolas G. Bazan ${ }^{1} \cdot$ Madigan M. Reid ${ }^{1} \cdot$ Valerie A. Cruz Flores ${ }^{1,2} \cdot$ Juan E. Gallo $^{3,4} \cdot$ William Lewis $^{1} \cdot$ Ludmila Belayev $^{1}$
}

Published online: 14 September 2021

(c) The Author(s) 2021

\begin{abstract}
Glioblastoma multiforme (GBM) is the most invasive type of glial tumor with poor overall survival, despite advances in surgical resection, chemotherapy, and radiation. One of the main challenges in treating GBM is related to the tumor's location, complex and heterogeneous biology, and high invasiveness. To meet the demand for oxygen and nutrients, growing tumors induce new blood vessels growth. Antibodies directed against vascular endothelial growth factor (VEGF), which promotes angiogenesis, have been developed to limit tumor growth. Bevacizumab (Avastin), an anti-VEGF monoclonal antibody, is the first approved angiogenesis inhibitor with therapeutic promise. However, it has limited efficacy, likely due to adaptive mutations in GBM, leading to overall survival compared to the standard of care in GBM patients. Molecular connections between angiogenesis, inflammation, oxidative stress pathways, and the development of gliomas have been recognized. Improvement in treatment outcomes for patients with GBM requires a multifaceted approach due to the converging dysregulation of signaling pathways. While most GBM clinical trials focus on "anti-angiogenic" modalities, stimulating inflammation resolution is a novel hostcentric therapeutic avenue. The selective therapeutic possibilities for targeting the tumor microenvironment, specifically angiogenic and inflammatory pathways expand. So, a combination of agents aiming to interfere with several mechanisms might be beneficial to improve outcomes. Our approach might also be combined with other therapies to enhance sustained effectiveness. Here, we discuss Suramab (anti-angiogenic), LAU-0901 (a platelet-activating factor receptor antagonist), Elovanoid (ELV; a novel lipid mediator), and their combination as potential alternatives to contain GBM growth and invasiveness.
\end{abstract}

Keywords Glioma $\cdot$ Suramin $\cdot$ Platelet-activating factor $\cdot$ Lipid mediators · Oncology

\section{Introduction}

Glioblastoma multiforme (GBM) is the most common and lethal intracranial malignancy, with a few advances in treatment over several decades. Standard-of-care therapy includes aggressive resection, radiation, and

Nicolas G. Bazan

nbazan@1suhsc.edu

1 Neuroscience Center of Excellence, School of Medicine, Louisiana State University Health New Orleans, New Orleans, LA, USA

2 Cancer and Blood Disorders Institute, Johns Hopkins All Children's Hospital, St. Petersburg, FL, USA

3 Instituto de Investigaciones en Medicina Translacional, Universidad Austral, CONICET, Buenos Aires, Argentina

4 Departamento de Oftalmología, Hospital Universitario Austral, Pilar, Buenos Aires, Argentina chemotherapy, but the median overall survival remains less than two years [1]. One of the challenges in the treatment of GBM is its aggressive growth characteristics. Complete surgical resection of the tumor is impossible because of infiltrative growth, multiple lesions, and microscopic spread. Thus, there is a strong need for new and effective GBM treatment. The molecular heterogeneity of GBM allows for adaptive mutations 
and drug resistance; thus, a multi-target approach is necessary targeting cells in the microenvironment. Non-transformed cells in this microhabitat are less susceptible to these adaptations, making them an ideal target. The microenvironment of glioblastoma harbors multiple cell types, which are believed to make distinct contributions to tumor progression and invasion [2] (Fig. 1). These cells include but are not limited to microglia, astrocytes, macrophages, pericytes, fibroblasts, and vascular cells. Gliomas are highly vascular tumors, and the endothelial cells, pericytes, and astrocytes that form the neurovascular unit function support tumor progression. In addition, microglia cells promote glioma migration and tumor growth [2]. Astrocytes can be converted into a reactive phenotype by the glioma microenvironment and secrete many factors that influence tumor growth [3]. The elements, pathways, and interactions provide a new perspective on the cell biology of brain tumors, which may ultimately generate a new treatment paradigm (Fig. 1).
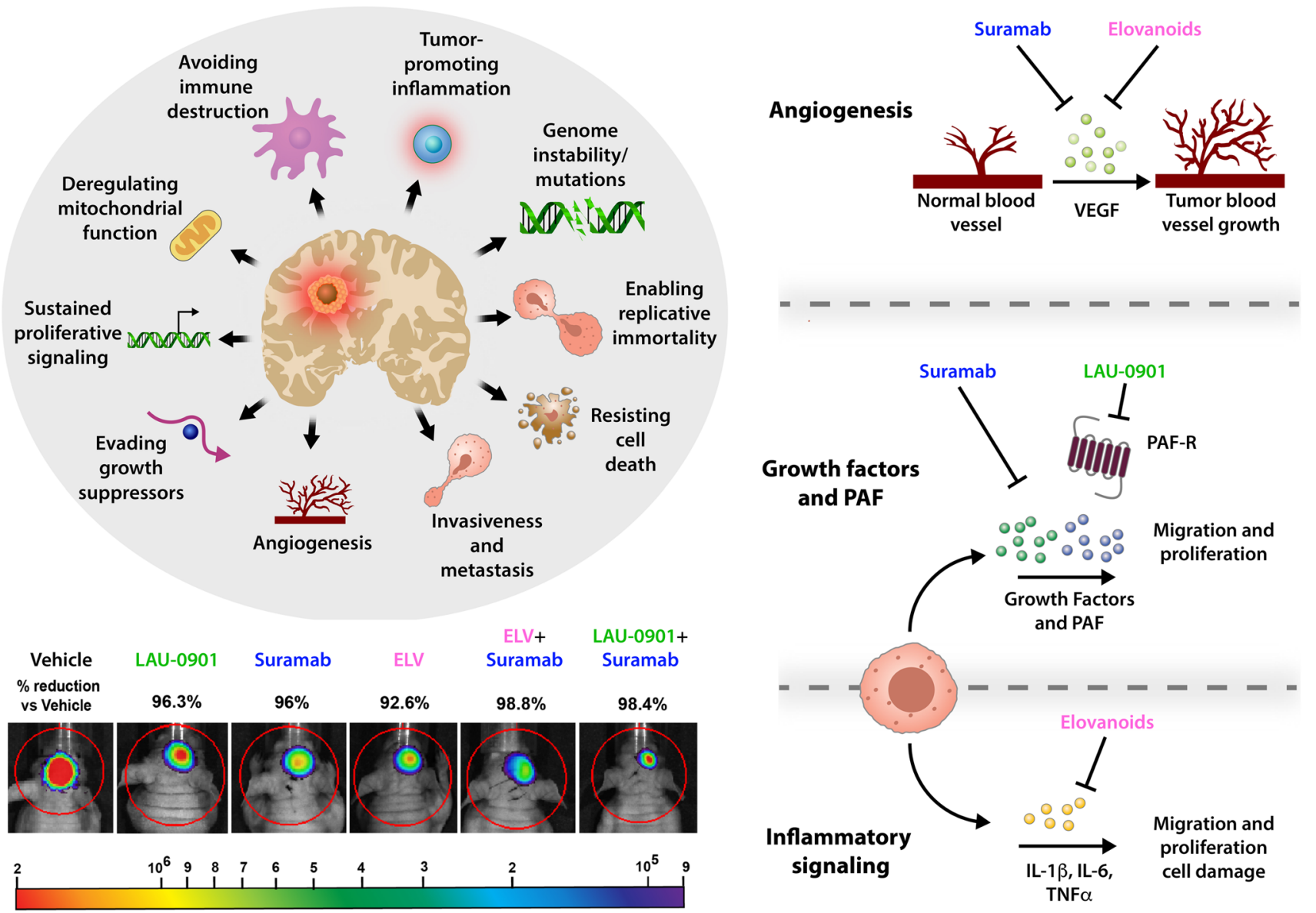

Luminescence $\left(\mathrm{p} / \mathrm{sec} / \mathrm{cm}^{2} / \mathrm{sr} \min =9.00 \mathrm{e} 4 \mathrm{max}=2.00 \mathrm{e} 6\right)$

Fig. 1 Diagram depicting characteristics of glioblastoma and potentially novel therapeutics. Glioblastomas comprise multiple cell types: microglia, astrocytes, fibroblasts, and endothelial cells facilitating tumor progression. Cytokines released by glioma cells recruit immune cells into the tumor microenvironment, inducing pro-inflammatory signaling. Inflammatory signaling elicits pro-tumor activity allowing cells to evade immune cells contributing to tumor progression. Increased growth factor and platelet-activating factor (PAF) secretion from surrounding and glioma cells and their ability to evade growth factor suppressors contribute to the tumor's proliferative and invasive nature. Vascular endothelial growth factor (VEGF) is a critical growth factor for blood vessel formation in glioblastoma. Inhibition of inflammatory signaling molecules IL-1 $\beta$, IL- 6 , and TNF $\alpha$ by elovanoids (ELVs) and inhibition of growth factor and PAF activity by Suramab and LAU-0901 reduces tumor proliferation and migration. Suramab and ELV also reduce blood vessel formation through inhibition of VEGF. Representative bioluminescent images of the brain tumors after implantation of the luciferase modified U87MG cells from all experimental groups on day 30 . The intensity of light emission corresponding to tumor burden is represented by a colorimetric scale, where red indicates the highest radiance and blue/violet shows the least. There was progressive and rapid tumor growth in the saline group. In contrast, mice treated with LAU-0901, Suramab, ELV, ELV + Suramab, and LAU-0901 + Suramab showed reduced tumor growth compared to vehicle-treated mice 


\section{Suramab (combination of Suramin plus Avastin) is a novel anti-angiogenic combination for GBM treatment}

Anti-angiogenic strategies for treating high-grade gliomas have a solid biologic rationale since these tumors produce large amounts of vascular endothelial growth factor (VEGF) and are highly vascular. Due to their high metabolic demands, growing solid tumors depends on vascularization to provide nutrients and oxygen and disposal of embolic waste products. Because VEGF is a critical growth factor required for new blood vessel formation, anti-VEGF agents were initially developed to block tumor growth by inhibiting blood vessel formation [4]. However, bevacizumab (brand name Avastin), a humanized monoclonal antibody developed to neutralize human VEGF, failed to improve survival benefit as monotherapy but conferred a survival benefit only in combination with chemotherapy or immuno- therapy [4]. A potential explanation for the success of combined treatments is that Avastin "normalizes" the abnormal vasculature of tumors, resulting in improved delivery of concurrently administered anticancer drugs, as well as alleviation of hypoxia.

Suramab is a new pharmaceutical combination of two anti-angiogenic compounds, suramin, and Avastin, which showed a high synergistic effect when administered jointly [5]. Suramin is a 100-year-old drug used to treat African sleeping sickness caused by Trypanosoma brucei rhodesiense [6]. It is a multifunctional molecule with many potential applications, from parasitic and viral diseases to cancer, snakebite, and autism. It has demonstrated anticancer activity by inhibiting the binding of multiple growth factors and glutamatergic synaptic transmission [6]. It has an extremely long half-life, but repetitive dosing results in drug accumulation [7]. Remarkably, a new pharmaceutical combination of suramin and Avastin, administrated at relatively low doses, has a tremendous anti-angiogenic effect, synergistic like, with greater intensity and longer duration than the effect produced by a mono-doses of Avastin or suramin [5]. It was demonstrated that Suramab strongly reduced tumor growth in colorectal carcinoma in mice and reduced neovascularization in a rabbit model of corneal angiogenesis [5]. Surprisingly, there are no studies to evaluate the efficacy of Suramab on GBM so far.

\section{LAU-0901 is a selective PAF-receptor antagonist and a potent inhibitor of inflammation and tumor growth}

In contrast to anti-angiogenic strategies, stimulation of inflammation resolution is a novel host-focused alternative to complement current therapies. The tumor microenvironment, primarily orchestrated by inflammatory molecules, promotes such tumors' proliferation, survival, and migration, and it seems logical to employ antiinflammatory drugs.

Described almost 50 years ago, the phospholipid mediator platelet-activating factor (PAF) has been implicated in many pathologic processes. PAF is a potent mediator of inflammation involved in inflammatory diseases such as atherosclerosis, cardiovascular diseases, and cancer [8]. PAF induces robust systemic pro-inflammatory, pro-proliferative, and delayed immune-suppressive responses via the activation of PAF receptor (PAF-R) which is implicated in various pathological conditions rationalizing its exploration in cancer development as many malignant cells express PAF-R [8]. Recent studies demonstrated the implication of PAF in cancer growth and metastasis [8]. Circulating or cancer cells synthesizes PAF and its presence in the tumor microenvironment. Inducible pathways that result in the development of tumor angiogenesis and metastasis may involve PAF binding on its receptor. Increased expression of tumoral PAF-R has been associated with invasiveness, increased tumor stages, tumor status, and poor prognosis in lung and esophageal squamous cell carcinoma [8]. Notably, patients who experienced decreased overall survival were found to have tumors expressing high levels of PAF-R compared to those with low tumor PAF-R expression [9]. The potential for inhibition of tumor growth and increased efficacy of other agents when targeting PAF-R has been proposed [8]. Reduction of tumor burden improved murine host survival, and the augmented efficacy of therapeutic agents has been observed using pharmacologic PAF-R antagonists [9]. Multiple structurally different but specific PAF-R antagonists have been shown to exert promising effects against experimental tumors [9]; however, these agents have yet to be explored in clinical settings. Thus, PAF may represent a rational therapeutic target in GBM. As a novel PAF-R antagonist, LAU-0901 has been previously shown to be neuroprotective in inflammation and ischemic stroke models [10]. LAU-0901 (2,4,6-trimethyl-1, 4-dihydro-pyridine-3, 5-dicarboxylic acid) is a selective PAF- $R$ antagonist and a potent inhibitor of inflammation response and apoptosis [10]. It has also been shown to exhibit neuroprotective bioactivity when applied to a model of focal cerebral ischemia in rats and mice [11].

\section{Elovanoids are a novel class of lipid mediators that regulate homeostasis}

Tumor growth is angiogenesis-dependent, and enhanced inflammation is a risk factor for many cancers. Inflammation is regulated by endogenous specialized pro-resolving lipid-autacoid 
mediators (SPMs). This includes resolvins, lipoxins, and protectins, which inhibit angiogenesis and mediate endogenous resolution by stimulating macrophage phagocytosis of cellular debris, resulting in reduced localized inflammatory cytokines $[12,13]$. Unlike the majority of anti-inflammatory agents, SPMs are non-immunosuppressive and non-toxic. It was demonstrated that pro-resolving lipid mediators and antiangiogenic therapy exhibit synergistic anti-tumor activity via resolvin receptor activation [14]. Notably, resolvins (RvD4 or RvD5) inhibited tumor growth at doses 10,000 times lower than anti-inflammatory agents such as aspirin and NSAIDs [14]. We recently discovered ELV, the novel class of endogenous pro-homeostatic lipid mediators that protect against excitotoxicity [15]. They are stereoselective mediators made on-demand and derived from very long-chain polyunsaturated fatty acids and have been shown to have a potent ability to inactivate pro-apoptotic and pro-inflammatory signaling in experimental stroke and neurodegenerative diseases [15].

In addition to anti-angiogenic and anti-inflammatory pathways, free fatty acid oxidation has been closely linked to GBM. Enhanced fatty acid oxidation provides glioblastoma cells metabolic plasticity to accommodate its dynamic nutrient microenvironment [16]. Thus, dynamic metabolic reprogramming plays a vital role during glioma genesis, which allows for the adaptation, survival, and proliferation of these cells in the diverse microenvironment implicit in this tumor. Thus, inhibition of fatty acid oxidation may provide an indirect approach to reduce tumor growth.

The development of effective GBM therapy presents challenges, one of which is the molecular heterogeneity and genetic instability of these tumors. To overcome this complexity, a multipronged approach that targets key signaling pathways, specifically angiogenesis, inflammation, and oxidative stress pathways, will open new therapeutic avenues.

Acknowledgements We thank LSUHSC Morphology and Imaging Core.

Author contribution NGB, JEG, and LB did conceptual design of commentary and experiment. MMR, VACF, WL, and LB drafted the article. MMR, WL, and GB prepared the graphical illustration with the assistance of artist illustrator Tom DiCesare; NGB and LB revised the article. All authors reviewed the submitted version of the manuscript. LB and NGB approved the final version of the manuscript on behalf of all authors.

Funding This project was supported by the EENT Foundation of New Orleans (NGB) and by Resident Research grants from LSUHSC Pediatrics Hematology-Oncology (VACF).

\section{Data availability N/A}

Code availability N/A

\section{Declarations}

Ethics approval All animal experiments were approved by the Institutional Animal Care and Use Committee of the Louisiana State University Health Sciences Center, New Orleans. All animal procedures were performed to minimize pain or discomfort in accordance with current protocols

\section{Consent to participate N/A}

\section{Consent for publication N/A}

Conflict of interest The subject matter of this manuscript is protected by a US provisional patent application assigned to the Board of Supervisors of Louisiana State University and Agricultural and Mechanical College (LSU) and licensed to a company, Neuresto Therapeutics, founded by co-author Nicolas G. Bazan.

Open Access This article is licensed under a Creative Commons Attribution 4.0 International License, which permits use, sharing, adaptation, distribution and reproduction in any medium or format, as long as you give appropriate credit to the original author(s) and the source, provide a link to the Creative Commons licence, and indicate if changes were made. The images or other third party material in this article are included in the article's Creative Commons licence, unless indicated otherwise in a credit line to the material. If material is not included in the article's Creative Commons licence and your intended use is not permitted by statutory regulation or exceeds the permitted use, you will need to obtain permission directly from the copyright holder. To view a copy of this licence, visit http://creativecommons.org/licenses/by/4.0/.

\section{References}

1. Tan, A. C., Ashley, D. M., López, G. Y., Malinzak, M., Friedman, H. S., \& Khasraw, M. (2020). Management of glioblastoma: state of the art and future directions. CA: A Cancer Journal for Clinicians, 70(4), 299-312. https://doi.org/10.3322/caac.21613

2. Charles, N. A., Holland, E. C., Gilbertson, R., Glass, R., \& Kettenmann, H. (2011). The brain tumor microenvironment. Glia, 59(8), 1169-1180. https://doi.org/10.1002/glia.21136

3. Fishbein, A., Hammock, B. D., Serhan, C. N., \& Panigrahy, D. (2021). Carcinogenesis: Failure of resolution of inflammation? Pharmacology \& Therapeutics, 218, 107670. https://doi.org/10. 1016/j.pharmthera.2020.107670

4. Garcia, J., Hurwitz, H. I., Sandler, A. B., Miles, D., Coleman, R. L., Deurloo, R., \& Chinot, O. L. (2020). Bevacizumab (Avastin $($ ) in cancer treatment: A review of 15 years of clinical experience and future outlook. Cancer Treatment Reviews, 86, 102017. https://doi.org/10.1016/j.ctrv.2020.102017

5. Lopez, E. S., Rizzo, M. M., Croxatto, J. O., Mazzolini, G., \& Gallo, J. E. (2011). Suramab, a novel anti-angiogenic agent, reduces tumor growth and corneal neovascularization. Cancer Chemotherapy and Pharmacology, 67(3), 723-728. https://doi. org/10.1007/s00280-010-1457-z

6. Wiedemar, N., Hauser, D. A., \& Mäser, P. (2020). 100 Years of Suramin. Antimicrobial Agents and Chemotherapy, 64(3), e01168-e1219. https://doi.org/10.1128/AAC.01168-19

7. Grossman, S. A., Phuphanich, S., Lesser, G., Rozental, J., Grochow, L. B., Fisher, J., \& Piantadosi, S. (2001). Toxicity, efficacy, and pharmacology of suramin in adults with recurrent highgrade gliomas. Journal of Clinical Oncology, 19(13), 3260-3266. https://doi.org/10.1200/JCO.2001.19.13.3260 
8. Tsoupras, A. B., Iatrou, C., Frangia, C., \& Demopoulos, C. A. (2009). The implication of platelet activating factor in cancer growth and metastasis: Potent beneficial role of PAF-inhibitors and antioxidants. Infectious Disorders Drug Targets, 9(4), 390399. https://doi.org/10.2174/187152609788922555

9. de Oliveira, S. I., Andrade, L. N., Onuchic, A. C., Nonogaki, S., Fernandes, P. D., Pinheiro, M. C., \& Jancar, S. (2010). Plateletactivating factor receptor (PAF-R)-dependent pathways control tumour growth and tumour response to chemotherapy. BMC Cancer, 10(1), 200. https://doi.org/10.1186/1471-2407-10-200

10. He, J., \& Bazan, H. E. P. (2006). Synergistic effect of plateletactivating factor and tumor necrosis factor-alpha on corneal myofibroblast apoptosis. Investigative Ophthalmology \& Visual Science, 47(3), 883-891. https://doi.org/10.1167/iovs.05-0581

11. Belayev, L., Khoutorova, L., Atkins, K., Gordon, W. C., AlvarezBuilla, J., \& Bazan, N. G. (2008). LAU-0901, a novel plateletactivating factor antagonist, is highly neuroprotective in cerebral ischemia. Experimental Neurology, 214(2), 253-258. https://doi. org/10.1016/j.expneurol.2008.08.009

12. Bazan, N. G., Eady, T. N., Khoutorova, L., Atkins, K. D., Hong, S., Lu, Y., \& Belayev, L. (2012). Novel aspirin-triggered neuroprotectin D1 attenuates cerebral ischemic injury after experimental stroke. Experimental Neurology, 236(1), 122-130. https://doi. org/10.1016/j.expneurol.2012.04.007

13. Panigrahy, D., Gilligan, M. M., Huang, S., Gartung, A., CortésPuch, I., Sime, P. J., \& Hammock, B. D. (2020). Inflammation resolution: A dual-pronged approach to averting cytokine storms in COVID-19? Cancer and Metastasis Reviews, 39(2), 337-340. https://doi.org/10.1007/s10555-020-09889-4

14. Sulciner, M. L., Serhan, C. N., Gilligan, M. M., Mudge, D. K., Chang, J., Gartung, A., \& Panigrahy, D. (2017). Resolvins suppress tumor growth and enhance cancer therapy. Journal of Experimental Medicine, 215(1), 115-140. https://doi.org/10.1084/jem. 20170681

15. Bhattacharjee, S., Jun, B., Belayev, L., Heap, J., Kautzmann, M.-A., Obenaus, A., \& Bazan, N. G. (2017). Elovanoids are a novel class of homeostatic lipid mediators that protect neural cell integrity upon injury. Science Advances, 3(9), e1700735. https:// doi.org/10.1126/sciadv.1700735

16. Kant, S., Kesarwani, P., Prabhu, A., Graham, S. F., Buelow, K. L., Nakano, I., \& Chinnaiyan, P. (2020). Enhanced fatty acid oxidation provides glioblastoma cells metabolic plasticity to accommodate to its dynamic nutrient microenvironment. Cell Death \& Disease, 11(4), 1-13. https://doi.org/10.1038/s41419-020-2449-5

Publisher's Note Springer Nature remains neutral with regard to jurisdictional claims in published maps and institutional affiliations. 